\title{
Democratizing Mobile App Development for Disaster Management
}

\begin{abstract}
Smartphones are being used for a wide range of activities including messaging, social networking, calendar and contact management as well as location and context-aware applications. The ubiquity of handheld computing technology has been found to be especially useful in disaster management and relief operations [Fajardo and Oppus, 2010]. Our focus is to enable developers to quickly deploy applications that take advantage of key sources that are fundamental for today's networked citizens, including Twitter feeds, Facebook posts, current news releases, and government data. These applications will also have the capability of empowering citizens involved in crisis situations to contribute via crowdsourcing, and to communicate upto-date information to others. We will leverage several technologies to develop this application framework, namely (i) Linked Data principles for structured data, (ii) existing data sources and ontologies for disaster management, and (iii) App Inventor, which is a mobile application development framework for non-programmers. In this paper, we describe our motivating use cases, our architecture, and our prototype implementation.
\end{abstract}

\section{Introduction}

Smartphones are quickly becoming the primary computing and communication platform for people's daily tasks. With the rise of social networks, "netizens" are now comfortable with frequently updating their social profiles with their current activities and/or locations. This new source of information, social signals from microblog platforms, has been found to be especially useful in disaster management and relief operations. For example, during the Beijing flash floods in July 2012, people took to Twitter to provide information about the flooded areas and collaboratively developed a live crisis map of the floods impact using Google Maps ${ }^{1}$. By integrating crowdsourced information with Geographic Information Systems (GIS) data or other open datasets released by the local

\footnotetext{
${ }^{1}$ http://iRevolution.net/2012/08/01/crisis-map-beijing-floods/
}

government, some technical volunteers have developed useful mobile applications for disaster reliefs. However, each organization usually has its own application that creates or consumes data stored in standalone databases, or even worse, in spreadsheets. This means most of these applications are built in silos without exploiting the potential of being "interlinked" with other data collected from other organizations, or even the government. The lack of expertise and the cost for building mobile applications cause relief workers to turn to more manual steps for merging various reports from volunteers. Consequently, the increasingly unorganized and scattered information become noise in the system and can sometimes slowdown the decision making process. We envision a framework that enables non-technical application developers to quickly build and deploy applications that easily reuse existing and crowdsourced structured information sources.

One major challenge in the afore-mentioned example is how to effectively integrate data generated by multiple parties, including the "crowd", relief organizations, and governement agencies. We address this challenge by using Linked Data principles ${ }^{2}$ to enable the reuse, extension and integration of heterogeneous structured data from distributed sources. Linked Data is a set of design principles proposed by the World Wide Web Consortium (W3C) for using Web technologies in order to support the distributed development of structured information such that it can be easily and automatically combined. However, consuming and generating Linked Data is difficult as pointed out in [Scharffe et al., 2012], especially on mobile devices. This implies that we need to also focus on reducing the barrier to the adoption of Linked Data technologies on smartphones.

This paper describes our ongoing work of extending the App Inventor ${ }^{3}$ platform with Linked Data technologies. App Inventor is an opensource app-building platform that allows users to drag-and-drop visual objects to create an application that can run on the Android system. We are developing "Linked Data" components for App Inventor that will allow app developers to easily build applications that explore and consume Linked Data datasets as well as publish structured data directly to remote Linked Data repositories. Our App Inventor components will focus on (i) embedding semantic con-

\footnotetext{
${ }^{2}$ http://www.w3.org/DesignIssues/LinkedData.html

${ }^{3} \mathrm{http} / / /$ appinventor.mit.edu/
} 


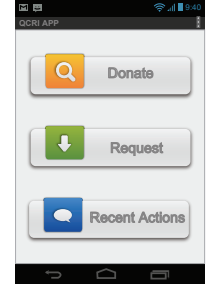

(a)

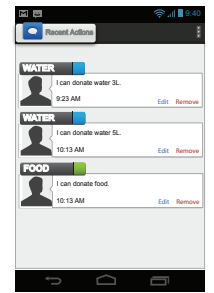

(c)

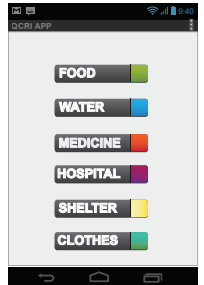

(b)

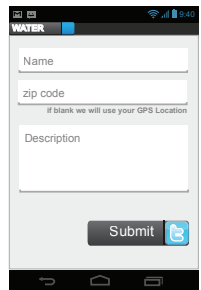

(d)

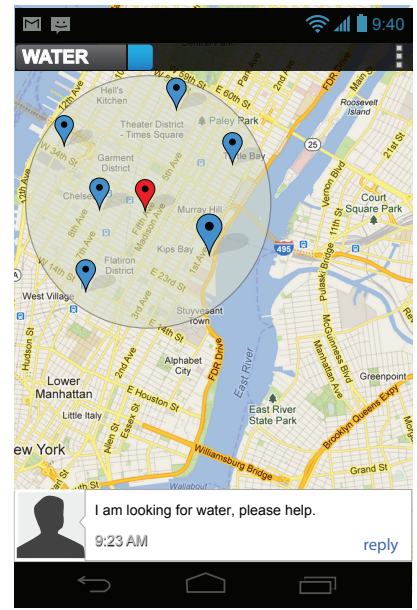

(e)

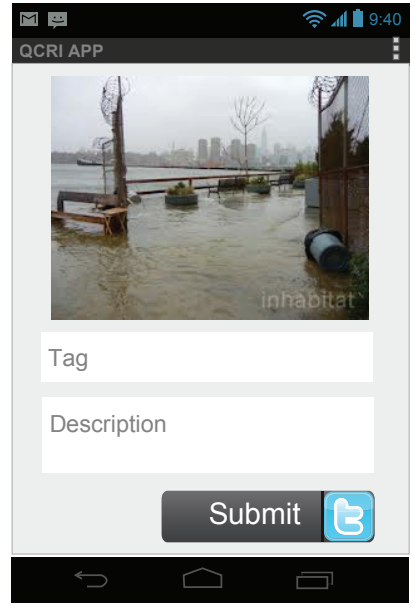

(f)

Figure 1: Two Semantic Web enabled mobile apps for disaster response situations

cepts directly into the process of mobile app-building while hiding most of the operational details from novice developers, and (ii) the integration of structured information from microblog platforms, crowdsourced and existing open data.

\section{Motivating scenarios}

We describe two applications that can potentially be used by people during a crisis situation: (1) App Donate-N-Request will be used to match requests and donations during a disaster scenario, and (2) App WeReport will be used to report scenes and information related to the disaster. These apps will have the ability to be integrated with different public datasets using linked data.

\subsection{Donate-N-Request App}

This app is inspired by MatchApp ${ }^{4}$, where requests for resources are met with the availability of those resources.

Consider this resource match-making scenario: Alice lives in New York City. After Hurricane Sandy hits, Alice wants to help people in need. Bob has been affected by the crisis. Both Bob, the requestor, and Alice, the donator, can place the donation and request for items within our app as shown in Figure 1(a) \& (b). They can also view/edit/remove recent list of their activities as shown in Figure 1(c). Alice chooses to donate water, so she checks up all the people within a 3 miles radius of her location who are in need of water on a map as shown in Figure 1(e). Bob had previously requested water by using our app (Figure 1(d)). Alice selects Bob from the list of people in need. Alice and Bob can message directly with each other to arrange the exchange.

\subsection{WeReport App}

On his way to the grocery store, after a severe hurricane, Joe noticed many parts around his community are in bad conditions. For example, fallen trees are blocking the road and

\footnotetext{
${ }^{4}$ http://iRevolution.net/2013/02/27/matchapp-disaster-responseapp/
}

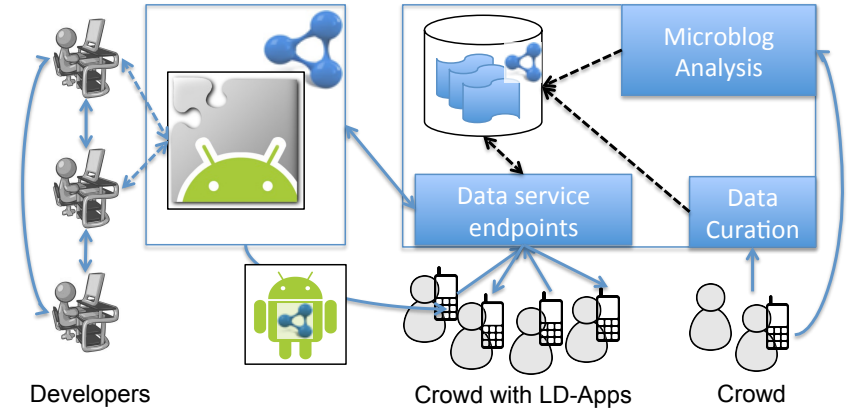

Figure 2: Architecture of Linked Data enabled crowdsourcing and application development plaform

some can be dangerous for the pedestrians. With our WeReport app, he could take either a picture or a video of the scene. He decides to take a picture of the road, by adding a "tag" and a "description" to the image as shown in Figure 1(f).

\section{Prototype Implementation}

In order to implement the apps described in Section 2, and gather the necessary data items, we explored the use of existing linked data vocabularies and tied them into App Inventor as illustrated in Figure 2.

\subsection{Linked Data}

Disaster management activities such co-ordinating relief operations, setting up shelters, reporting structural damages, and overseeing volunteers generate a lot of data. These need to be integrated and be interoperable with data provided by various organizations to be useful in decision making processes during disasters. We identified two vocabularies that are useful in modeling such data as Linked Data. They have varying degrees of support for some of the disaster concepts, and we used terms from both the vocabularies in our implementation. We discuss some shortcomings of these ontologies in 
Section 4.

\section{Humanitarian eXchange Language Situation and Response Standard (HXL)}

HXL $^{5}$ was developed through an initiative by the United $\mathrm{Na}$ tions High Commissioner for Refugees (UNHCR) after inspecting a broad range of systems in use with different humanitarian organizations. The schema is available in five main sections, namely: geolocation (information on the locations affected), humanitarian profile (information on the populations affected), metadata (who is collecting the data, date/time the data was collected, etc), response (information on the organization responding), and situation (information related to the emergency).

\section{Management Of A Crisis Vocabulary (MOAC)}

MOAC $^{6}$ was created after the earthquake in Haiti in 2010. Much of the focus in developing this vocabulary was to mitigate the inconsistencies between aggregated data and requirements of relief organizations. This was the first Linked Data vocabulary that had the objective of facilitating non-experts to provide data in Linked Data formats through crowd sourcing mechanisms such as Google Map Maker, Open Street Map, Twitter and Ushahidi. MOAC defines three main sections that relate to relief agency standards.

\subsection{App Inventor}

App Inventor provides a Web-based development platform for novices with no prior programming experience to create mobile applications easily. A user can drag and drop graphical objects on the interface editor (Figure 3(a)) to design an app or implement behaviors with blocks as shown in Figure 3(b). App Inventor also provides many high-level components for using smartphone resources as well as communicating with external web resources. For example, it has components for reading the GPS location sensor, taking photos with the camera, receiving SMS texts, and sending Twitter messages. By hiding most of the technical details, App Inventor gives its users the benefits to focus on designing app behaviors rather than lower-level debugging. However, App Inventor currently has little support for integrating application data with backend datastores, for example, to save application data as Linked Data or integrate with other Linked Data resources.

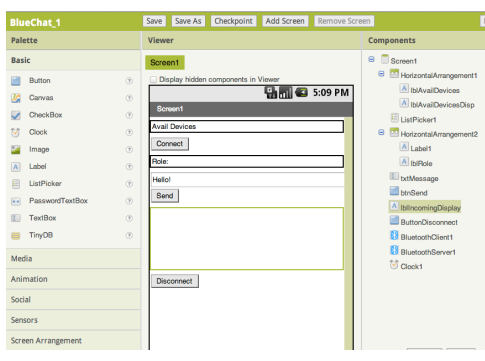

(a)

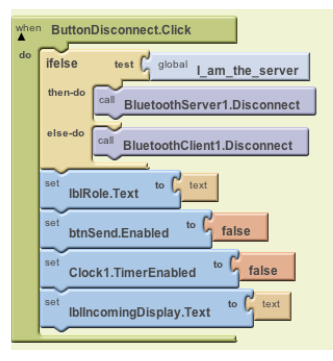

(b)
Figure 3: Interface of the App Inventor platform

\footnotetext{
${ }^{5}$ http://hxl.humanitarianresponse.info/ns/index.html

${ }^{6} \mathrm{http} / / /$ observedchange.com/moac/ns/
}

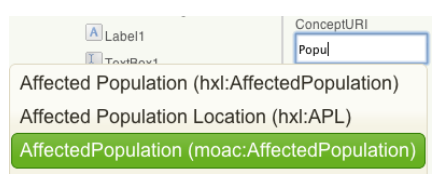

Figure 4: App Inventor autocompletion of concepts and properties powered by SPARQL queries over cached semantic web ontologies

\subsection{Linked Data Extension of App Inventor}

In order to enable developers using the App Inventor platform to interact with Linked Data sources, we provide an extended version of App Inventor with additional components and user interface elements powered by a modified version of the Jena framework [Carroll et al., 2004]. In this section we outline how we expose Linked Data concepts to application developers in the App Inventor the new components that can be used by developers to consume and generate Linked Data.

\section{Exploring Linked Data}

One challenge of using Linked Data is finding details of an ontology that describes a dataset. App developers should not be burdened with knowing all the details of an ontology. For example, when building a crisis response app, a developer may begin with a general concept such as Shelter and let the system provide more relevant information. Our extension provides an autocompletion capability in the designer interface to assist developers in selecting the appropriate ontological term (see Figure 4). We provide a service within the App Inventor that loads ontologies, indexes labels for classes and properties, and answers queries to support this autocompletion widget.

\section{Consuming Linked Data with Semantic Web Component}

The Semantic Web Component converts semantic forms into RDF graphs, executes and process results of SPARQL queries, and saves and loads the content of ontologies. For example, the app developer can execute a SPARQL query to fetch information about nearby shelters based on the user's current GPS location and show the results on a map.

\section{Publishing Linked Data with Semantic Form}

The Semantic Form is a layout component that can be used by developers to identify a collection of fields that should be applied to a particular ontological concept. Forms can also assign an autogenerated subject URI based on one or more fields to create new Linked Data instances. For example, a developer can have two textfields for the name and the description of a shelter. Semantic Form will automatically generate Linked Data triples if those fields are tagged with ontological terms like the properties shelterName and shelterDescription from the MOAC ontology.

\section{Discussion}

Typical types of data gathered and consumed during disaster situations includes: (1) Data describing response and resource characteristics (locations of people in evacuation 
zones, shelter locations); (2) Data on the scope and emerging effects (how many people are affected by for e.g. a hurricane, what populations are affected based on the new projected path of the hurricane); (3) Data on the outages of a disaster response and missing items to match with the relief capacity (how many shelters are in need of food rations, how much do they need, how much is in transit, etc); (4) Data to guide the recovery and construction efforts as the crisis nears the end (status of homes owned by shelter inhabitants). Although the existing disaster management vocabularies have support for many disaster management scenarios described above, we identified some shortcomings in them. For instance, they do not have comprehensive support for in-kind donations. HXL defines administrative units that may accept the donations, and the affected populations that would receive those donations. However there is no support to specify what the donation is, i.e. whether it is money, clothes, food, etc, as well as who is donating it and other logistics related to the donation. Similarly, MOAC has support for specifying the needs of a specific shelter and who is handling what in the WhoWhatWhere (W3) section of the vocabulary. However, there is nothing to describe data relating to donations. Another shortcoming of these two vocabularies is the lack of support for situational awareness data. As an example, if someone is reporting structural damage to a bridge that might be affecting a population, there is no way to link the bridge data to MOAC and/or HXL.

To address these shortcomings we are in the process of formulating a vocabulary that will tie in these disaster vocabularies with other related linked data vocabularies, e.g. Geonames, Semantically Interlinked Online Communities $(\mathrm{SIOC})^{7}$, etc. Geonames can be used to model geo-location information, and the related population information. SIOC can be used to tie in information available in microblog posts from tweets and Facebook posts after a disaster such as zones that people need to evacuate from, new developments in a disaster (e.g. the potential sighting of a looting), donation pledges along with requests for donations, etc.

With the proliferation of linked datasets, developers using App Inventor may want to use these data directly via App Inventor components without having to write SPARQL queries themselves. In order to facilitate this feature, we are in the process of developing "data wrapper" components. These components have built-in SPARQL queries to access frequently accessed data, with extended functionality to plugin custom SPARQL queries to get at more specific data items. With this functionality, we hope not to clutter the App Inventor component interface with data items that a majority of app inventor developers may not use while providing the flexibility to retrieve any kind of data the developer desires. As an example, consider a data wrapper component for the NYC demographics statistics datasets ${ }^{8}$. This dataset includes all the population statistics such as number of males and females as well as number of people based on their ethnic categorization in a given zipcode area. This ethnic categorization in-

\footnotetext{
${ }^{7} \mathrm{http} / / /$ sioc-project.org

${ }^{8} \mathrm{https}: / /$ nycopendata.socrata.com/Statistics/DemographicStatistics-By-Zip-Code
}

cludes generic categories such as white, black, hispanic, asian in addition to very specific categories such as pacific islander, asian non-hispanic, white non-hispanic, white hispanic, black non-hispanic, black hispanic, etc. The data wrapper would expose the gender based information and the general ethnicities in the interface with built-in SPARQL queries that could just be dragged and dropped on to the interface, whereas very specific queries to obtain information about a particular ethnicity (for e.g. black hispanic) could be written by the app inventor developer and plugged into the component.

Using linked data within a mobile environment has proved useful and challenging in other research. David et al. [2010] propose a general framework in order to introduce Linked Data sources as device content in the Android platform; D'Aquin et al. [2010] consume selected data sources and create new social link between people in the author's university; Razzak et al. [2010] utilize government data to support tourism within the author's city. However in all these approaches the application is domain specific and cannot be extended to be used with different data sources. Extending the App Inventor framework with Linked data will allow developers to be able to reuse available linked data sources and to tailor and extend apps to a variety of scenarios.

\section{Summary}

In this paper we have demonstrated the effective use of Linked Data within mobile applications with a special focus on disaster management. As organizations continue to expose their data as Linked Open Data, our platform will be able to help developers using the Linked Data App Inventor platform to come up with useful applications quickly and without much effort to help those in need during disasters.

\section{References}

[Carroll et al., 2004] Jeremy J. Carroll, Ian Dickinson, Chris Dollin, Dave Reynolds, Andy Seaborne, and Kevin Wilkinson. Jena: Implementing the semantic web recommendations. In Proceedings of the 13th international World Wide Web conference, pages 74-83, New York, NY, USA, 2004. ACM.

[d'Aquin et al., 2010] Mathieu d'Aquin, Fouad Zablith, and Enrico Motta. wayOU - linked data-based social location tracking in a large, distributed organisation. 2010.

[David and Euzenat, 2010] Jérôme David and Jérôme Euzenat. Linked data from your pocket: The android rdfcontentprovider. In ISWC 2010, 2010.

[Fajardo and Oppus, 2010] Jovilyn Therese B. Fajardo and Carlos M. Oppus. A mobile disaster management system using the android technology. WSEAS TRANSACTIONS on COMMUNICATIONS, 9, June 2010.

[Razzak et al., 2010] Faisal Razzak, Dario Bonino, and Fulvio Corno. Mobile interaction with smart environments through linked data. In IEEE International Conference on Systems Man. and Cybernetics, 2010.

[Scharffe et al., 2012] François Scharffe, Ghislain Atemezing, Raphaël Troncy, Fabien Gandon, Serena Villata, Bénédicte Bucher, Fayçal Hamdi, Laurent Bihanic, Gabriel Képéklian, Franck Cotton, et al. Enabling linked-data publication with the datalift platform. 2012. 